\title{
Differential Cross Sections for Higgs Boson Production at Tevatron Collider Energies
}

\author{
Edmond L. Berger $1, *$ and Jianwei Qiu ${ }^{2, t}$ \\ ${ }^{1}$ High Energy Physics Division, Argonne National Laboratory, Argonne, IL 60439 \\ ${ }^{2}$ Department of Physics and Astronomy, \\ Iowa State University, Ames, IA 50011
}

(Dated: December 17, 2018)

\begin{abstract}
The transverse momentum $Q_{T}$ distribution is computed for inclusive Higgs boson production at $\sqrt{S}=1.96 \mathrm{TeV}$. We include all-orders resummation of large logarithms associated with emission of soft gluons at small $Q_{T}$. We provide results for Higgs boson and $Z^{*}$ masses from $M_{Z}$ to $200 \mathrm{GeV}$. The relatively hard transverse momentum distribution for Higgs boson production suggests possibilities for improvement of the signal to background ratio.
\end{abstract}

*e-mail: berger@anl.gov

$\dagger$ †-mail: jwq@iastate.edu 


\section{INTRODUCTION}

Elucidation of the dynamics responsible for the breaking of electroweak symmetry is one of the primary goals in particle physics during this decade. In the current run-II of the Fermilab Tevatron, the upgraded Collider Detector Facility (CDF) and D0 detectors will search for the neutral Higgs boson $(h)$, the vehicle of symmetry breaking in the standard model (SM), as well as the Higgs bosons of the minimal supersymmetric standard model (MSSM). The task is challenging. The production cross sections and branching fractions into channels favorable for detection are relatively small, and the backgrounds from competing processes are large [1].

The fully inclusive gluon-gluon fusion subprocess [2, 3] $g g \rightarrow h X$ supplies the largest cross section at Tevatron energies. Its rate is a factor four or so greater than that of the next most important subprocess, associated production $q \bar{q} \rightarrow h V X$, where $V=W, Z$. In the region of modest Higgs boson masses, $m_{h}<135 \mathrm{GeV}$, the combination of gluon fusion production with the dominant decay process $h \rightarrow b \bar{b}$ should be attractive, but its promise is compromised by the overwhelming production of $b \bar{b}$ pairs from strong interactions background processes. For $m_{h}>135 \mathrm{GeV}$, the channel $h \rightarrow W W^{*}$ becomes dominant, and gluon fusion followed by $h \rightarrow W W^{*}$ provides a potential discovery mode at the Tevatron.

Calculations of the expected differential cross sections for production of the signal and backgrounds are important for evaluation of the required measurement accuracies and detector performance. Estimations of the expected transverse transverse momentum distributions can suggest selections in this variable that should improve background rejection. In this paper, we concentrate on $g g \rightarrow h X$ and discuss the behavior of the Higgs boson transverse momentum distribution in the region of small and intermediate values of $Q_{T}$. We expect our predictions to be directly pertinent in the region $m_{h}>135 \mathrm{GeV}$, but we also entertain optimism that experimenters may find clever ways to make use of the dominant channel $g g \rightarrow h X$ in the region $m_{h}<135 \mathrm{GeV}$.

In the gluon fusion process, production occurs through triangle loops of colored (s)particles that couple to the Higgs boson and to gluons. In the SM, the most relevant contribution is from a loop of top $(t)$ quarks. The coupling of gluons is simplified in the limit of large top quark mass $m_{t}[4]$. The $m_{t} \rightarrow \infty$ approximation is valid to an accuracy of $\sim 5 \%$ for $m_{h} \leq 2 m_{t}[5]$. Within this approach, the total cross section for $g g \rightarrow h X$, is known 
to next-to-next-to-leading order (NNLO) accuracy [6, 7]. The large $m_{t}$ approximation serves well also for the transverse momentum distribution when $m_{h}<2 m_{t}$ and the Higgs boson transverse momentum $Q_{T}$ is less than $m_{t}$ [8]. The next-to-leading order contributions are computed in Refs. [9, 10, 11].

When $Q_{T}$ is comparable to $m_{h}$, there is only one hard momentum scale in the perturbative expansion of the cross section as a function of the strong coupling $\alpha_{s}$, and fixed-order computations in perturbative QCD are expected to be applicable. However, in the region $Q_{T} \ll m_{h}$, where the cross section is greatest, the coefficients of the expansion in $\alpha_{s}$ depend functionally on logarithms of the ratio of the two quantities, $m_{h}$ and $Q_{T},\left(\alpha_{s} / \pi\right) \ln ^{2}\left(m_{h}^{2} / Q_{T}^{2}\right)$. The relevant expansion parameter in the perturbative series is close to 1 , and straightforward fixed-order perturbation theory is inapplicable for $Q_{T} \ll m_{h}$. All-orders resummation is the established method for mastering the large logarithmic coefficients of the expansion in $\alpha_{s}$ and for obtaining well-behaved cross sections at intermediate and small $Q_{T}$ 12, 13, 14]. Using renormalization group techniques, Collins, Soper, and Sterman (CSS) 13] devised a $b$ space resummation formalism that resums all logarithmic terms as singular as $\left(1 / Q_{T}^{2}\right) \ln ^{n}\left(m_{h}^{2} / Q_{T}^{2}\right)$ when $Q_{T} \rightarrow 0$. This formalism has been used widely for computations of the transverse momentum distributions of Higgs bosons [15, 16, 17, 18, 19, 20, 21] and other processes.

In this paper, we use the $b$ space resummation approach discussed in some detail in our Ref. 20] to derive predictions for the $Q_{T}$ spectrum of Higgs boson production at the Fermilab Tevatron energy $\sqrt{S}=1.96 \mathrm{TeV}$. We resum the large logarithmic terms associated with soft gluon emission to all orders in $\alpha_{s}$ obtaining well defined predictions for the full range of $Q_{T}$. We employ expressions for the parton-level hard-scattering functions valid through firstorder in $\alpha_{s}$, including contributions from the glue-glue, quark-glue, and quark-antiquark incident partonic subprocesses. We present differential cross sections for masses of the Higgs boson that span the range of present interest in the SM, from $m_{h}=M_{Z}=91.187 \mathrm{GeV}$ to $m_{h}=200 \mathrm{GeV}$. To illustrate interesting differences, we also provide results for the $Z$ boson. In this paper, we also show the integrated $Q_{T}$ distributions $\sigma\left(Q_{T}>Q_{T m i n}\right)$ for rapidity within the interval $|y|<1.0$. This distribution indicates immediately what fraction of the Higgs boson cross section may be sacrificed if a selection is made on $Q_{T}$ for background rejection.

Our predictions are presented and discussed in Sec. II. Conclusions and a discussion of background rejection are found in Sec. III. 


\section{PREDICTIONS}

Our predictions are based on the formalism described in detail in our Ref. [20]. For the perturbative $A_{g}$ and $B_{g}$ functions in the Sudakov factor, we make an expansion valid through second order $(n=2)$ in the strong coupling $\alpha_{s}$. For the short-distance coefficient functions $C_{a \rightarrow b}$ used to compute the modified parton densities, we retain the expansion through $n=1$, i.e., to first order in $\alpha_{s}$. We remark that the functions $A_{g}, B_{g}$, and $C_{a \rightarrow b}$ have well behaved perturbative expansions, free of large logarithmic terms. We use a next-toleading order form for $\alpha_{s}(\mu)$ and next-to-leading order normal parton densities $\phi(x, \mu)$ [22]. In the fixed-order perturbative expressions that enter the " $Y$ " function, we select a fixed renormalization/factorization scale $\mu=\kappa \sqrt{m_{h}^{2}+Q_{T}^{2}}$, with $\kappa=0.5$. For the resummed term, we take as our central value $\mu=c / b$, with $c=2 e^{-\gamma_{E}} \approx 1$. We examine sensitivity to the choice of $\mu$ by computing cross sections with other choices of this scale. The extrapolation into the non-perturbative region of large $b$ is accomplished with the form devised by Qiu and Zhang [23].

In Fig. 1, we show the predicted differential $Q_{T}$ distributions. We present results for three choices of mass of the Higgs boson, $m_{h}=M_{Z}=91.187 \mathrm{GeV}, m_{h}=125 \mathrm{GeV}$, and $m_{h}=150 \mathrm{GeV}$ (where the $W W^{*}$ decay channel is dominant). In all cases, the solid lines represent the predictions of resummation at next-to-leading order (NLO) accuracy and, for comparison, the dashed lines show the results of resummation at leading-order (LO) accuracy [24].

Two points are evident in the comparison of $Z$ boson and Higgs boson production, with $m_{h}=M_{Z}$, Fig. 1(c) and (d). The peak in the $Q_{T}$ distribution occurs at a smaller value of $Q_{T}$ for $Z$ production. At $y=0$, the curve peaks at $Q_{T} \sim 3.0 \mathrm{GeV}$ for $Z$ production and at $Q_{T} \sim 7.3 \mathrm{GeV}$ for Higgs boson production. Second, the distribution is narrower for $Z$ production, falling to half its maximum by $Q_{T} \sim 9.0 \mathrm{GeV}$, whereas the half-maximum for Higgs production is not reached until $Q_{T} \sim 21 \mathrm{GeV}$. The physics behind these important differences is that the larger QCD color factors produce more gluonic showering in the glue-glue scattering subprocess that dominates inclusive Higgs boson production than in the fermionic subprocesses relevant for $Z$ production. After all-orders resummation, the enhanced showering suppresses the large- $b\left(\right.$ small $\left.Q_{T}\right)$ region more effectively for Higgs boson production. 

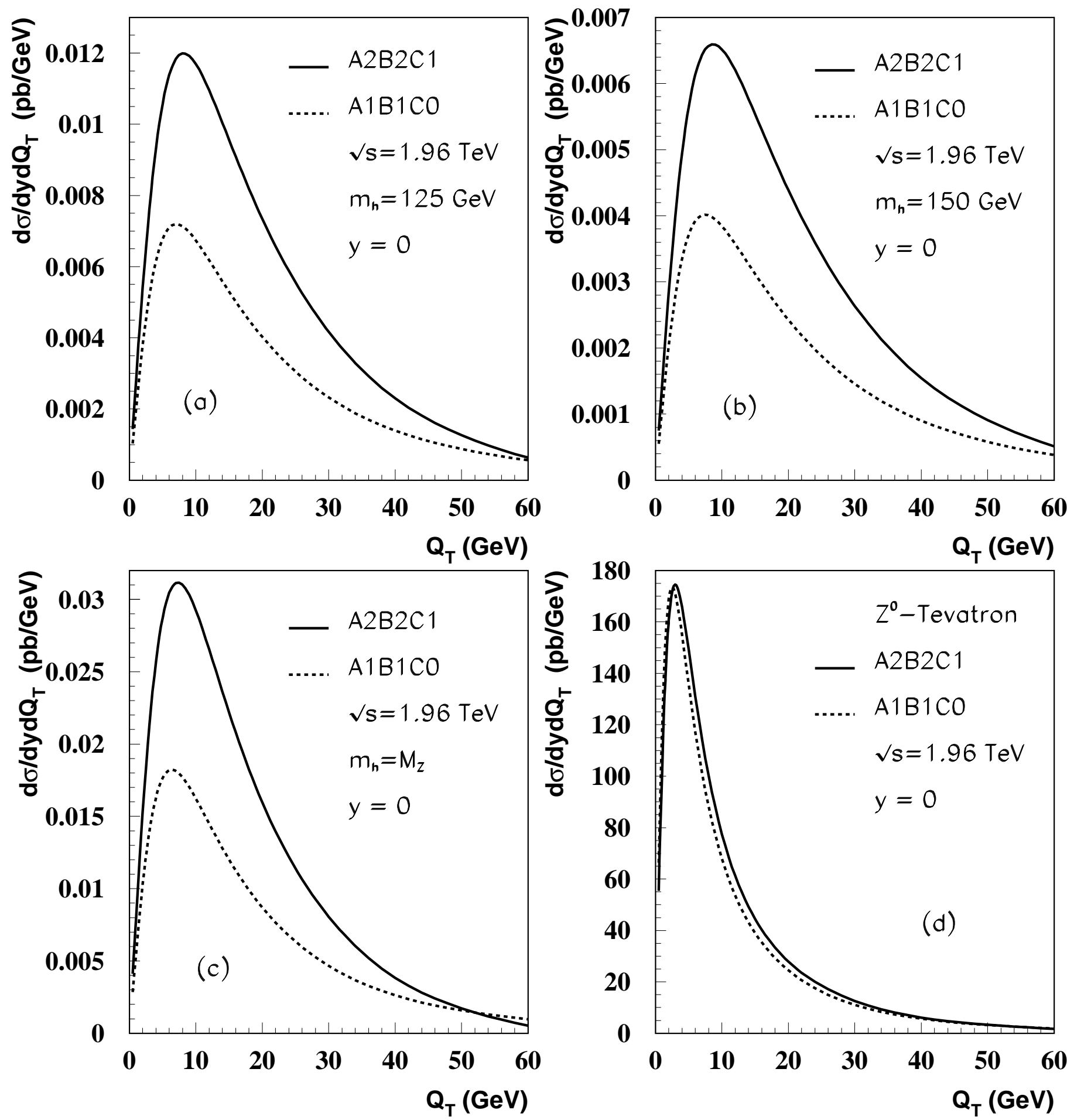

FIG. 1: Differential cross sections for Higgs boson production at $\sqrt{S}=1.96$ TeV at (a) $Q=m_{h}=$ $125 \mathrm{GeV}$, (b) $Q=m_{h}=150 \mathrm{GeV}$, and (c) $Q=M_{Z}$. The NLO prediction is shown as a solid line and the LO prediction as a dashed line. In (d) we show analogous results for $Z$ boson production. 
For Higgs boson production, there is a substantial difference in the predictions at NLO and at $\mathrm{LO}$, but the differences are slight for $Z$ production. The role of the $C$ function is responsible for these effects. The first two coefficients in the expansion of $C$ are published 17 , 18, 19]. In powers of $\left(\alpha_{s} / \pi\right)$, the $C^{(1)}$ coefficients for the $g g$ and $q \bar{q}$ cases are

$$
\begin{aligned}
& C_{g \rightarrow g}^{(1)}(z)=\delta(1-z) \frac{1}{2}\left[C_{A}\left(\frac{2 \pi^{2}+11}{6}\right)+\frac{\pi^{2}}{6} C_{A}\right] . \\
& C_{q \rightarrow q}^{(1)}(z)=\delta(1-z) \frac{1}{2}\left[C_{F}\left(\frac{2 \pi^{2}-24}{6}\right)+\frac{\pi^{2}}{6} C_{F}\right]+\frac{1}{2} C_{F}(1-z) .
\end{aligned}
$$

In the $q \bar{q}$ case relevant for $Z$ production, there is a strong cancellation, $2 \pi^{2}-24$, reducing the effect of the NLO contribution. For Higgs boson production, the analogous term is additive, $2 \pi^{2}+11$. The constant terms -24 and 11 , respectively, are associated with virtual corrections at NLO and can be traced to the interference between the tree and one-loop diagrams. The $2 \pi^{2}-24$ term is effectively the finite piece of the one-loop correction to the $q \bar{q} \rightarrow Z$ vertex while the $2 \pi^{2}+11$ term results from the finite piece of the one-loop correction to the effective $g g \rightarrow h$ vertex.

Integrating our $Q_{T}$ distributions over all $Q_{T}$ and rapidity $y$, we obtain the total cross sections as a function of Higgs boson mass shown in Fig. 2] We may compare these cross sections to the values listed in Table 3 of Ravindran et al [7] based on their fully inclusive calculation, without resummation. Their results are obtained with different parton densities and are quoted at $\sqrt{S}=2 \mathrm{TeV}$, making the comparison somewhat imprecise. The integral of our NLO resummed cross section lies between the NLO and NNLO inclusive results, slightly above the NLO values, for all masses shown, as would be expected qualitatively. Our calculation includes the ingredients $A_{g}^{(1)}$ and $B_{g}^{(1)}$ of a NLO calculation with, in addition, other ingredients such as $A_{g}^{(2)}$ of the NNLO total cross section.

Comparison of the predicted $Q_{T}$ distributions for Higgs boson production at different masses of the Higgs boson shows that the peak of the distribution shifts to greater $Q_{T}$ as $m_{h}$ grows and that the distribution broadens somewhat. At $y=0$, the peaks are centered at about $Q_{T}=7.3,8.2,8.7$, and $9.5 \mathrm{GeV}$ for $m_{h}=M_{Z}, 125,150$, and $200 \mathrm{GeV}$, respectively. The change of the $Q_{T}$ distribution with $m_{h}$ can be examined quantitatively with plots of the mean value $\left\langle Q_{T}>\right.$ and of the root-mean-square $\left\langle Q_{T}^{2}>^{\frac{1}{2}}\right.$. We find that $\left\langle Q_{T}>\right.$ grows from about $19 \mathrm{GeV}$ at $m_{h}=M_{Z}$ to about $28 \mathrm{GeV}$ at $m_{h}=200 \mathrm{GeV}$. The curve may be approximated with a straight line over this range, with $<Q_{T}>\simeq 0.079 m_{h}+12 \mathrm{GeV}$. The 


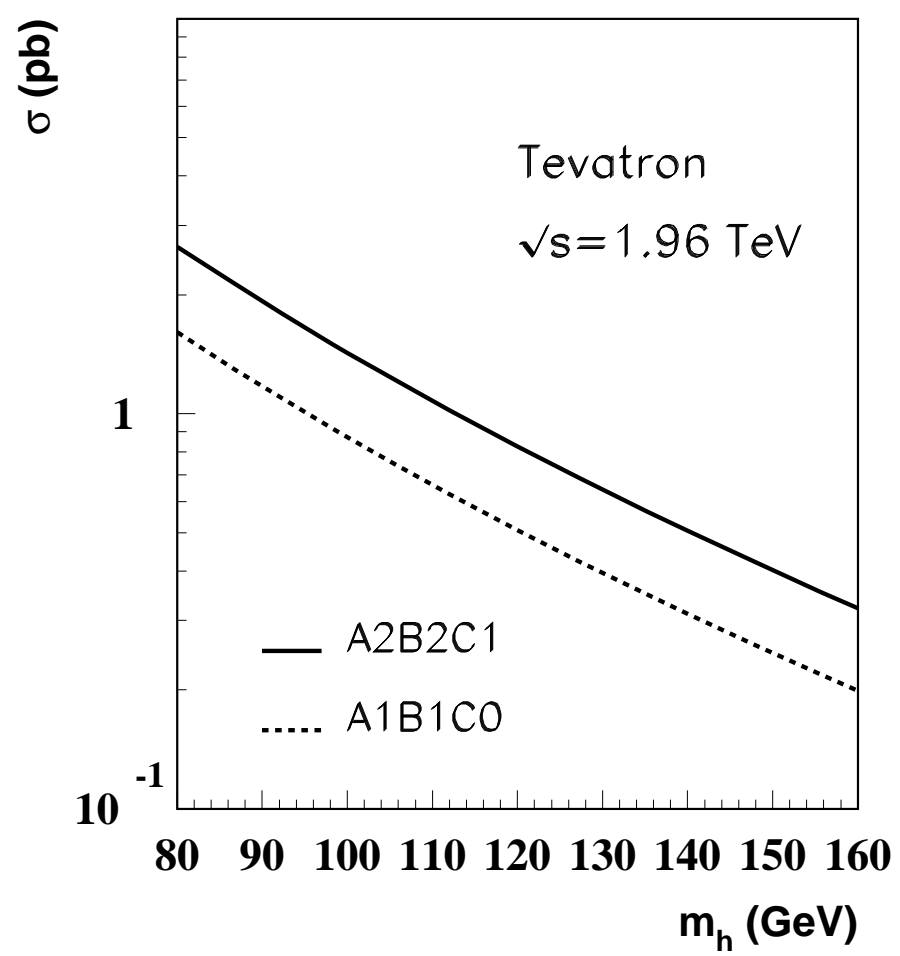

FIG. 2: The integral of the resummed cross section over all $Q_{T}$ and rapidity for Higgs boson production at $\sqrt{S}=1.96 \mathrm{TeV}$ as a function of Higgs boson mass at NLO (solid line) and LO (dashed line) accuracy.

root-mean-square of the distribution grows from about $27 \mathrm{GeV}$ to about $38 \mathrm{GeV}$, reflecting the broadening of the $Q_{T}$ distribution with $m_{h}$.

For comparison with Higgs boson production, we quote our predictions for $Z$ production: $<Q_{T}>=13 \mathrm{GeV}$ and $<Q_{T}^{2}>^{1 / 2}=20 \mathrm{GeV}$. The difference $<Q_{T}^{h}>-<Q_{T}^{Z}>\simeq 6 \mathrm{GeV}$ at $m_{h}=M_{Z}$ is a manifestation of more significant gluonic radiation in Higgs boson production.

In anticipation of a discussion of selections on $Q_{T}$ to improve signal to background ratios, it is instructive to examine production of $Z^{*}$ bosons, with mass greater than $M_{Z}$. For $Z^{*}$ masses above $M_{Z}$ we assume that the dominant $q \bar{q}$ production model is unchanged except for the difference in mass of the $Z^{*}$. We find that the change in the $Q_{T}$ distribution with mass is much less significant than is seen for the Higgs boson. For example, the peak position at $y=0$ increases to only $3.4 \mathrm{GeV}$ at $M_{Z^{*}}=200 \mathrm{GeV}$ from its value of $3 \mathrm{GeV}$ at $M_{Z}$.

We introduce a new distribution for Higgs boson production that represents the integral of the differential cross section for all $Q_{T}$ greater than a minimum value, and for rapidity integrated over the interval $|y|<1.0$. The results are shown in Fig. 3. At $Q_{\text {Tmin }}=10 \mathrm{GeV}$, 

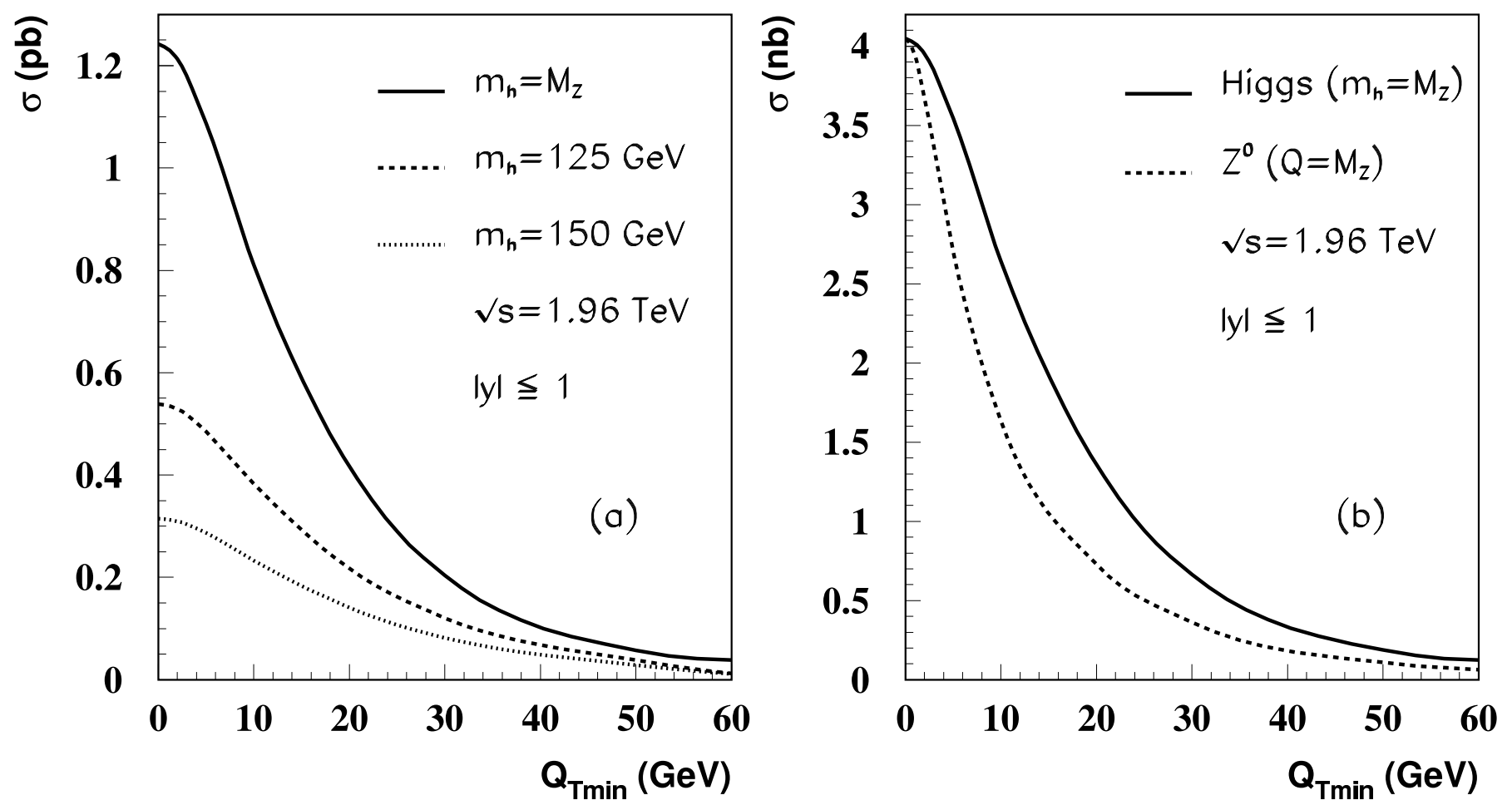

FIG. 3: (a) Cross sections for Higgs boson production at $\sqrt{S}=1.96$ TeV integrated over the ranges $Q_{T}>Q_{T m i n}$ and $|y|<1.0$ for three values of the mass of the Higgs boson: $m_{h}=125$ GeV, 150 $G e V$, and $m_{h}=M_{Z}$. In (b), we show analogous results for $Z$ boson production and, for ease of comparison, the curve for Higgs boson production at $m_{h}=M_{Z}$ rescaled so that the cross section at $Q_{T \min }=0$ is the same as that for the $Z$ case.

the integrated distribution has dropped to $40 \%$ of its total for $Z$ production but to only $65 \%, 71 \%$, and $74 \%$ for Higgs boson production at $m_{h}=M_{Z}, 125 \mathrm{GeV}$, and $150 \mathrm{GeV}$, respectively. The irreducible background for Higgs boson decay to pairs of $W$ 's arise from the QCD annihilation subprocess $q \bar{q} \rightarrow W W$. This subprocess has the same initial state structure as $Z\left(Z^{*}\right)$ boson production. The harder $Q_{T}$ spectrum for the glue-glue dominated Higgs boson production shows that the signal to background ratio can be enhanced if Higgs bosons are selected with large $Q_{T}$.

Choices of parameters are made in obtaining our results, including the renormalization/factorization scale $\mu$ and the non-perturbative input. Our default value $\mu=c / b$ with $c=2 e^{-\gamma_{E}} \approx 1$ provides a scale that varies inversely with the impact parameter $b$. This selection has the virtue that logarithmic dependence on $\mu$ is removed from the coefficient functions $C$ [13, 20]. The integration in $b$ space is dominated by the region near the peak in 
the $b$ distribution, $b \simeq 0.08$ for $m_{h}=125 \mathrm{GeV}$. Using the conjugate relationship $Q_{T} \sim 1 / b$, we note that the typical hard scale $\mu$ is therefore $<\mu>\simeq 12.5 \mathrm{GeV}\left(\simeq 0.1 m_{h}\right)$. To examine sensitivity to the selection of $\mu$, we consider other choices that are independent of $b$. Taking a value as large as $\mu=2 m_{h}$ produces changes in the magnitude of $d \sigma / d y d Q_{T}$ at the peak position of no more than about $20 \%$, much less than the difference between the NLO and LO results in Fig. 1. Uncertainties associated with non-perturbative physics in the region of large $b$ are at most 1 to $2 \%$ depending on the size of the power corrections [23].

\section{CONCLUSIONS}

Discovery of the Higgs particle is essential to shed light on the mechanism of electroweak symmetry breaking. The partonic subprocess $g+g \rightarrow h X$ dominates Higgs boson production in hadronic reactions when the Higgs boson mass is in the expected range $m_{h}<200 \mathrm{GeV}$. The two-scale nature of the production dynamics, with mass $m_{h}$ and transverse momentum $Q_{T}$ both potentially large, and the fact that the fixed-order perturbative QCD contributions are singular as $Q_{T} \rightarrow 0$, necessitates all-orders resummation of large logarithmic contributions in order to obtain meaningful predictions for the $Q_{T}$ distribution particularly in the region of modest $Q_{T}$ where the cross section is largest. We perform this resummation of multiple soft-gluon emission using an impact parameter $b$-space formalism. The resummed $Q_{T}$ distributions are determined primarily by the perturbatively calculated $b$-space distributions at small $b$, with negligible contributions from the non-perturbative region of large $b$.

In this paper, we present predictions for the $Q_{T}$ distributions of Higgs boson and $Z$ production at $\sqrt{S}=1.96 \mathrm{TeV}$. At the same mass, $m_{h}=M_{Z}$, the predicted mean value $<Q_{T}>$ is about $6 \mathrm{GeV}$ larger for Higgs boson production than for $Z$ boson production. For the Higgs boson, $\left\langle Q_{T}>\right.$ grows from about $19 \mathrm{GeV}$ at $m_{h}=M_{Z}$ to about $28 \mathrm{GeV}$ at $m_{h}=200 \mathrm{GeV}$, and the root-mean-square $<Q_{T}^{2}>^{1 / 2}$ from about $27 \mathrm{GeV}$ to about $38 \mathrm{GeV}$.

Searches for the Higgs boson in its decay to $W W$ require an excellent understanding of the production characteristics of both the signal and backgrounds. The irreducible backgrounds arise from the QCD annihilation subprocess $q \bar{q} \rightarrow W W$. Of interest to us is the expected $Q_{T}$ dependence of the ratio of signal to irreducible background. In this paper we provide predictions for the transverse momentum distribution of the signal. The annihila- 
tion subprocess $q \bar{q} \rightarrow W W$ has the same initial state structure as $Z\left(Z^{*}\right)$ boson production. As we show, the $Q_{T}$ spectrum of $Z^{*}$ production is predicted to be significantly softer than that for Higgs boson production. We suggest therefore that a selection of events with large $Q_{T}^{W W}$ should help in improving the signal to background ratio. The placement of the cut requires, of course, appropriate optimization to maintain signal significance. In the mass range $m_{h}<135 \mathrm{GeV}$ where $h \rightarrow b \bar{b}$ is the leading decay mode, the QCD subprocess $g g \rightarrow b \bar{b}$ supplies the dominant background. Since the signal and background are both produced from $g g$ scattering, we expect them to have very similar $Q_{T}$ dependences at $m_{h}=m_{b \bar{b}}$, and means other than selections on $Q_{T}$ will be necessary for background suppression in this mass interval.

\section{ACKNOWLEDGMENTS}

Research in the High Energy Physics Division at Argonne is supported by the United States Department of Energy, Division of High Energy Physics, under Contract W-31-109ENG-38. JWQ is supported in part by the United States Department of Energy under Grant No. DE-FG02-87ER40371.

[1] M. Carena et al., "Report of the Tevatron Higgs working group," arXiv:hep-ph/0010338.

[2] F. Wilczek, Phys. Rev. Lett. 39, 1304 (1977); H. M. Georgi, S. L. Glashow, M. E. Machacek, and D. V. Nanopoulos, Phys. Rev. Lett. 40, 692 (1978); J. R. Ellis, M. K. Gaillard, D. V. Nanopoulos, and C. T. Sachrajda, Phys. Lett. B 83, 339 (1979); T. G. Rizzo, Phys. Rev. D 22, 178 (1980) [Addendum-ibid. D 22, 1824 (1980)].

[3] D. Graudenz, M. Spira, and P. M. Zerwas, Phys. Rev. Lett. 70, 1372 (1993); M. Spira, A. Djouadi, D. Graudenz, and P. M. Zerwas, Nucl. Phys. B 453, 17 (1995) hep-ph/9504378.

[4] J. R. Ellis, M. K. Gaillard, and D. V. Nanopoulos, Nucl. Phys. B 106, 292 (1976); M.A. Shifman, A. I. Vainshtein, M. B. Voloshin, and V. I. Zakharov, Sov. J. Nucl. Phys. 30, 711 (1979) [Yad. Fiz. 30, 1368 (1979)].

[5] S. Dawson, Nucl. Phys. B 359, 283 (1991); A. Djouadi, M. Spira, and P. M. Zerwas, Phys. Lett. B 264, 440 (1991). 
[6] R. V. Harlander and W. B. Kilgore, Phys. Rev. Lett. 88, 201801 (2002) arXiv:hep-ph/0201206; C. Anastasiou and K. Melnikov, Nucl. Phys. B646, 220 (2002) arXiv:hep-ph/0207004;

[7] V. Ravindran, J. Smith, and W. L. van Neerven, arXiv:hep-ph/0302135.

[8] R. K. Ellis, I. Hinchliffe, M. Soldate, and J. J. van der Bij, Nucl. Phys. B 297, 221 (1988); U. Baur and E. W. Glover, Nucl. Phys. B 339, 38 (1990); R. P. Kauffman, Phys. Rev. D 45, 1512 (1992); S. Dawson and R. P. Kauffman, Phys. Rev. Lett. 68, 2273 (1992); R. P. Kauffman, S. V. Desai, and D. Risal, Phys. Rev. D 55, 4005 (1997) [Erratum-ibid. D 58, 119901 (1998)] arXiv:hep-ph/9610541; V. Del Duca, W. Kilgore, C. Oleari, C. Schmidt, and D. Zeppenfeld, Phys. Rev. Lett. 87, 122001 (2001) arXiv:hep-ph/0105129; Nucl. Phys. B 616, 367 (2001) arXiv:hep-ph/0108030.

[9] D. de Florian, M. Grazzini, and Z. Kunszt, Phys. Rev. Lett. 82, 5209 (1999) arXiv:hep-ph/9902483.

[10] V. Ravindran, J. Smith, and W. L. Van Neerven, Nucl. Phys. B 634, 247 (2002) arXiv:hep-ph/0201114.

[11] C. J. Glosser and C. R. Schmidt, arXiv:hep-ph/0209248.

[12] J. C. Collins and D. E. Soper, Nucl. Phys. B 193, 381 (1981) [Erratum-ibid. B 213, 545 (1983)]; Nucl. Phys. B 197, 446 (1982).

[13] J. C. Collins, D. E. Soper, and G. Sterman, Nucl. Phys. B 250, 199 (1985).

[14] R. K. Ellis and S. Veseli, Nucl. Phys. B 511, 649 (1998) arXiv:hep-ph/9706526; R. K. Ellis, D. A. Ross, and S. Veseli, Nucl. Phys. B 503, 309 (1997) arXiv:hep-ph/9704239.

[15] S. Catani, E. D’Emilio, and L. Trentadue, Phys. Lett. B 211, 335 (1988).

[16] I. Hinchliffe and S. F. Novaes, Phys. Rev. D 38, 3475 (1988).

[17] R. P. Kauffman, Phys. Rev. D 44, 1415 (1991); R. P. Kauffman, Phys. Rev. D 45, 1512 (1992).

[18] C.-P. Yuan, Phys. Lett. B 283, 395 (1992); C. Balazs and C.-P. Yuan, Phys. Lett. B 478, 192 (2000) arXiv:hep-ph/0001103; C. Balazs, J. Huston, and I. Puljak, Phys. Rev. D 63, 014021 (2001) arXiv:hep-ph/0002032.

[19] D. de Florian and M. Grazzini, Phys. Rev. Lett. 85, 4678 (2000) arXiv:hep-ph/0008152; Nucl. Phys. B 616, 247 (2001) arXiv:hep-ph/0108273; S. Catani, D. de Florian, and M. Grazzini, Nucl. Phys. B 596, 299 (2001) arXiv:hep-ph/0008184.

[20] E. L. Berger and J. W. Qiu, Phys. Rev. D 67, 034026 (2003) arXiv:hep-ph/0210135. 
[21] G. Bozzi, S. Catani, D. de Florian and M. Grazzini, arXiv:hep-ph/0302104.

[22] CTEQ Collaboration, H. L. Lai et al., Eur. Phys. J. C 12, 375 (2000) arXiv:hep-ph/9903282.

[23] J. W. Qiu and X. F. Zhang, Phys. Rev. D 63, 114011 (2001) arXiv:hep-ph/0012348; Phys. Rev. Lett. 86, 2724 (2001) arXiv:hep-ph/0012058.

[24] We use the common but not necessarily universal terminology whereby resummation at nextto-leading order (NLO) accuracy means keeping terms through order $\alpha_{s}^{2}$ in the expansions of the functions $A_{g}$ and $B_{g}$ and through order $\alpha_{s}$ in the expansion of the the function $C$. Resummation at leading-order (LO) accuracy means retaining terms through order $\alpha_{s}$ in the expansions of $A_{g}$ and $B_{g}$ and through order $\alpha_{s}^{0}$ in the expansion of $C$. 